\begin{abstract}
RES'LRICTED COS'T FUNCTIONS AND THE
RATE OF REITURN TO QUASI-FIXED FACTORS, WITH AN APPLICATION TO R\&D AND CAPITAL

IN THE BELI, SYSTEM
\end{abstract}

Mark Schankerman

M. Ishaq Nadiri

Working Paper No. 1259
NATIONAL BUREAU OF ECONOMIC RESEARCH
1050 Massachusetts Avenue
Cambridge, MA 02138 January 1984

The research reported here is part of the NBER's research program in Productivity. Any opinions expressed are those of the authors and not those of the National Bureau of Economic Research. 
NBER Working Paper \#1259

January 1984

\title{
Restricted Cost Functions and the Rate of Return To Quasi-Fixed Factors, with an Application to R\&D and Capital in the Bell System
}

\begin{abstract}
This paper provides a statistical test to assess the adequacy of static equilibrium models. The test is based on a restricted cost function framework together with the envelope conditions which characterize static equilibrium for the quasi-fixed factors. We also show how restricted cost function models can be exploited to investigate some important issues such as the calculation of the rates of return to quasi-fixed factors, the determination of overor underinvestment in particular assets, and the distinction between short run excess capacity and long run economies of scale. We provide an empirical application of these techniques to data on the Bell System for the period 1947-1976, treating the stocks of physical capital and of research and development (R\&D) as quasifixed inputs. The results suggest that there was substantial overinvestment in capital and underinvestment in R\&D compared to the static equilibrium levels, and that the rates of return to capital and R\&D were about 4.5 and $10-15$ percent, respectively.
\end{abstract}

Dr. Mark Schankerman

National Bureau of Economic Research 269 Mercer Street

8 th Floor

New York, NY 10003
Dr. M. Ishaq Nadiri Department of Economics New York University 15-19 West 4th Street New York, NY 10012 


\section{Introduction}

A number of papers have appeared recently on the specification and estimation of dynamic equilibrium models of factor demand and their application to the explanation of productivity growth. ${ }^{1}$ These papers represent a significant departure from earlier empirical work in this area, which was based on static equilibrium models and dynamic models with ad hoc adjustment mechanisms. The dynamic equilibrium models begin with the theory of restricted cost functions, in which the firm minimizes the costs of production over a set of completely variable inputs conditional on a given stock of quasi-fixed factors (hereafter, fixed factors). ${ }^{2}$ The assumption is made that the firm faces smooth, convex costs of adjusting the stocks of fixed factors and an intertemporal cost minimization problem is solved to obtain explicit time paths for the fixed inputs. These models yield many useful insights, especially in terms of the dynamic pattern of input substitution and complementarity, but they make stringent demands on the available data and have some conceptual limitations. The assumption of smooth convex adjustment costs rules out potentially interesting asymmetries regarding the costs of investment and disinvestment. Moreover, there may be various reasons for a divergence between the actual level and static equilibrium level of fixed factors which cannot be summarized adequately by smooth convex adjustment costs, such as regulatory restrictions, credit rationing and other institutional rigidities. One can embrace the idea of dynamic equilibrium models and yet maintain that the particular formulation should reflect the problem under study. An alternative approach which sidesteps these complications is to use a restricted cost (or profit) function framework but leave the levels 
of the fixed factors unexplained. These restricted equilibrium models are more flexible in that they do not impose a particular structure on the evolution of fixed factors, but for the same reason they yield no information about dynamic substitution possibilities or the time path of fixed inputs. This approach has been applied widely to analyze the short and long run structure (but not the dynamic path) of factor demand and costs (see papers in Berndt and Field 1981; also Lau and Yotopolous 1971), to study productivity growth (Caves, Christensen and Swanson 1981), and to estimate the divergence between the observed and static equilibrium levels of the fixed inputs (Brown and Christensen 1981). In a recent important paper, Berndt and Fuss (1982) show explicitly how measured growth in total factor productivity reflects both shifts in the production possibility frontier and any existing divergences of the fixed inputs from their static equilibrium levels, and they demonstrate that the correct adjustment for this problem requires information on the shadow prices of the fixed inputs. Given the focus of many of these studies, it is a natural extension and would be very useful to develop an empirical test of the divergence of fixed inputs from the static equilibrium levels and a method to estimate their shadow prices directly. Unfortunately, such a test is not yet available in the literature.

The first objective of this paper is to provide a usable empirical test of the hypothesis that fixed inputs are at their static equilibrium levels. In a restricted cost function framework, the static equilibrium level of a fixed factor is defined by the well-known envelope condition which equates the marginal savings in variable costs and the market rental price of the fixed input (Samuelson 1953; Gorman 1968). We formulate a 
statistical test of whether the envelope conditions are validated by the data. The test procedure should assist the empirical researcher in choosing between the static, restricted and dynamic equilibrium modelling strategies. We also show how to retrieve information about the long run structure of costs and input substitution from the restricted cost function. The second objective is to provide a method of estimating the marginal rates of return to the fixed inputs from a restricted cost function. Aside from their intrinsic interest, we suggest how estimates of the rates of return (or the shadow prices to which they are related) can be used to implement the proposal by Berndt and Fuss (1982) to adjust measured productivity growth for departures of fixed factors from their static equilibrium levels.

Finally, we provide an empirical application of these procedures to data on the Bell System for the period 1947-1976, in which the stocks of physical capital and of research and development (R\&D) are treated as fixed inputs. Summarizing briefly, the empirical results indicate that the hypothesis of static equilibrium can be rejected strongly for capital and (more ambiguously) for R\&D, that there was excessive investment in capital and underinvestment in $R \& D$ compared to the static equilibrium levels, and that the marginal rates of return to capital and R\&D were on the order of 4.5 and 10-15 percent, respectively.

The paper is organized as follows. Section 1 sets out the restricted cost function framework and shows how to retrieve characteristics of the underlying long run technology. The procedure for testing the envelope conditions is developed in Section 2. The method of estimating the rates of return to fixed inputs is presented in Section 3. Section 4 provides 
an empirical application of the model to the Bell System. Concluding remarks follow.

\section{Methodological Framework}

Let $T(y, x, z)=0$ denote the underlying transformation function connecting the level of output $y$, a set of variable inputs $x=\left(x_{1}, \ldots, x_{m}\right)$, and a set of fixed inputs $z=\left(z_{1}, \ldots, z_{n}\right)$. If $T(\cdot)$ satisfies certain regularity conditions (Lau 1976) and the firm minimizes the variable costs of producing $y$, conditional on the vector $z$ and fixed prices for variable inputs $p$, there exists a restricted (or variable) cost function

$$
\mathrm{VC}=\mathrm{F}(\mathrm{y}, \mathrm{p} ; \mathrm{z}) \text {. }
$$

The function $F(y, p ; z)$ is monotonically nondecreasing and concave in $p$, nondecreasing in $y$, and nonfncreasing and convex in $z$. By Hotelling's Lemma the set of conditional demand functions for the variable factors is obtained by differentiating $F(\cdot)$ with respect to variable input prices:

$$
x=F_{p}(y, p ; z)
$$

where a subscript denotes partial differentiation so $\mathrm{F}_{\mathrm{p}}=\left(\mathrm{F}_{\mathrm{p}_{1}}, \ldots, \mathrm{F}_{\mathrm{p}_{\mathrm{m}}}\right)$. The associated short run cost function includes the costs of fixed factors:

$$
S C=F(y, p ; z)+r z^{\prime}
$$

where a prime denotes a transpose of a vector, and $r=\left(r_{1}, \ldots, r_{n}\right)$ is a vector of market rental (service) prices of the fixed inputs. 
The preceding functions are defined for-arbitrary positive levels of z. The static equilibrium levels of $z, z^{*}$, are defined implicitly by the envelope conditions (Samuelson 1953; Gorman 1968):

$$
-F_{z}\left(y, P ; z^{*}\right)=r
$$

The left hand side of (4) represents the marginal reduction in variable costs due to $z$, or the shadow price of $z$. The envelope condition says that fixed factors are at the static equilibrium levels if and only if their shadow prices equal the market rental prices. Also note for future reference that (3) and (4) imply that $\partial \mathrm{SC} / \partial \mathrm{z}_{\mathrm{J}}=0$ if and only if the envelope condition for $z_{j}$ holds, 1.e., $z_{j}=z_{j}^{*}$. Finally, let $z^{*}=H(y, p, r)$ denote the demand functions for $z$ implied by (4) and substitute into (3) to obtain the long run cost function

$$
C=F(y, p, H(y, p, r))+r H^{\prime}(y, p, r)=G(y, p, r)
$$

where the prime represents a transpose of the vector.

The duality theorems which link transformation and restricted cost functions guarantee that the structure of production can be inferred from the restricted equilibrium framework (see for example Lau 1976). The structure of production is summarized by the long run output elasticity of costs and the partial elasticities of substitution, and we now show how to derive these measures from the restricted cost function.

Let $n_{v}=\partial \ln V C / \partial \ln y$ and $n_{s}=\partial \ln S C / \partial \ln y$ denote the output elasticity of variable and short run (total) costs, respectively. Differentiating (2) with respect to output and letting $z$ vary arbitrarily, we obtain.

$$
S C_{y}=F_{y}+F_{z} z_{y}^{\prime}+r Z_{y}^{\prime}
$$


To allow for Inoptimal choice of $z$, let $-F_{z}=r+d$ where $d=\left(d_{1}, \ldots, d_{n}\right)$ denotes the deviations of the shadow prices from the market rental prices of $z$. Using this notation and converting (6) to elasticities we obtain

$$
n_{s}=(1+\pi)^{-1}\left[n_{v}-\pi_{d} n_{z y}^{\prime}\right]
$$

where $\pi=\left(1+r z^{\prime} / v c\right), \pi_{d}=d z^{\prime} / v c$ and $n_{z y}=\partial \ln z / \partial \ln y$. Therefore, the short run (total) cost elasticity reflects the varlable cost elasticity. the divergence of fixed inputs from their static equilibrium levels, and the response of those inputs to changes in the level of output. If static equilibrium holds, $z=z^{*}$ and $d=0$, short and long run costs coincide, and the long run cost elasticity $n=\partial \ln C / \partial$ ln $y$ becomes

$$
n_{\ell}=\left(1+\pi^{*}\right)^{-1} n_{v}^{*}
$$

where $\pi^{*}$ and $n_{v}^{*}$ are evaluated at $z=z^{*}$. As noted by Hanoch (1975), the proper measure of scale economies is given by $n_{\ell}^{-1}$. It is important to note that the long run cost elasticity can be retrleved from the variable cost elasticity only in the nefghborhood of the static equilibrium levels of fixed factors. That is, equation (8) holds only at $z=z^{*}$ and inferences based on (8) are Invalid if $z \neq z^{*}$. This finding contrasts with Caves, Christensen and Swanson (1981) who claim to show that the long run scale economies can be retrleved at any arbitrary level of $\mathrm{z}^{3}$

The analysis also 1llustrates the classic problem of disentangling the effects of "excess capacity" and economies of scale (Borts 1960). Note from (7) and (8) that

$$
n_{\ell}-n_{s}=\left(1+\pi^{*}\right)^{-1}\left[\left(n_{v}^{*}-n_{v}\right)+(1+\pi)^{-1} n_{v}+\left(\frac{1+\pi^{*}}{1+\pi}\right) \pi_{d} n_{z y}^{\prime}\right]
$$


Assume for simplicity that the restricted cost function is homogeneous in output, so $n_{v}$ is a constant and $n_{v}^{*}=n_{v}$. Suppose that excess stocks of fixed factors are held (i.e., $z>z$, which implies $d>0$ and $\pi_{d}>0$ ) and that those inputs are normal $\left(n_{z y}>0\right)$. Then it follows from (9) that $n_{\ell}>n_{s^{*}}$ In this case, incorrectly using observed (short run) data on $z$ and equation (8) to infer $\mathrm{n}_{\ell}$ would yield an underestimate of the true $\mathrm{n}_{\ell}$ and hence an overstatement of returns to scale. The contrary holds if $z<z^{*}$. The correct procedure to infer $n_{\ell}$ from $n_{v}$ is either to test the hypothesis $z=z^{*}$ and then (conditional on nonrejection) evaluate (8) at the observed $z$, or to estimate $z^{\star}$ as those values which solve the envelope conditions and use them to evaluate equation (8). We return to this point later.

Consider now the Allen partial elasticities of substitution (AES). We limit the technical derivation to a brief summary and provide the intuition behind the results. A complete technical derivation is presented in Brown and Christensen (1981). Let $\sigma_{v}^{i j}$ and $\sigma_{l}^{i j}$ denote the variable and long run AES between inputs $i$ and $j$, and $z^{k}=H^{k}(y, P, r)$ be the demand function for $z^{k}$ implied by the envelope conditions ( 3 ). The AES can be expressed in terms of the restricted and total cost functions as (Uzawa 1962):

$$
\begin{aligned}
& \sigma_{v}^{i j}=F F_{i j} / F_{i} F_{j} \\
& \sigma_{\mathcal{i j}}^{i j}=G G_{i j} / G_{i} G_{j}
\end{aligned}
$$

where $F(\cdot)$ and $G(\cdot)$ are the restricted and long run cost functions given in (1) and (5), superscripts denote inputs, and subscripts denote partial derivatives with respect to input prices unless otherwise noted. of 
course, $\sigma_{v}^{1 f}$ is defined only for variable inputs since fixed factors are given in the short run. For clarity we partition inputs into a set of variable factors (VF) and a set of fixed factors (FF), with associated indices $i, j \in V F$ and $k, m \in F F$.

The expressions for the $\sigma_{\ell}$ are obtained by taking the derivations required in (10b) and using the envelope conditions (4) to simplify them. The procedure is straightforward, except perhaps to note that in differentiating $G(y, p, r)$ with respect to prices of fixed inputs the dependence of $z$ on those prices, as shown in (5), must be recognized. The results can be summarized as follows:

$$
\begin{aligned}
& \sigma_{\ell}^{1 j}=G\left(F_{i j}+\sum_{k} F_{j z} k^{H_{1}^{k}}\right) / F_{i} F_{j} \\
& \sigma_{\ell}^{k i}=G\left(\underset{k}{\sum_{j z} F_{1}^{H k}}\right) / H^{k} F_{i} \\
& \sigma_{\ell}^{k m}=G H_{m}^{k} / H^{k} H^{m}
\end{aligned}
$$

where $F_{j z} k$ is the cross partial of $F(\cdot)$ with respect to $p_{j}$ and $z^{k}$. Equations $(11 a-11 c)$ provide the long run AES between variable factors, between variable and fixed factors, and between fixed factors, respectively. Using (10a) we can also write $(11 a)$ as $\sigma^{1 j}=\left(1+\pi^{\star}\right)\left[\sigma_{v}^{1 j}+F\left(\underset{k}{F z} k_{i}{ }_{i}^{k}\right) / F_{i} F_{j}\right]$ where $\pi^{*}$ was defined earlier. This form of the long run AES allows a simple intuitive interpretation. The $\sigma_{l}^{i f}$ consists of a direct and an indirect effect of $p_{i}$ on $x_{j}$. The term $\sigma_{v}^{i j}$ represents the direct effect, holding $z$ constant in the short run. The second group of terms in the brackets represents the indirect effect of $p_{1}$ on $x_{j}$ via the effect of $p_{i}$ on the optimal levels of the fixed factors $z^{k}$. To see this, recall that 
$H_{i}^{k}=\partial z^{k} / \partial p_{i}$ and note that by Shephard's Lemma $F_{j z k}=\partial x_{j} / \partial z^{k}$. Hence, $\sum_{k} F_{j z} k_{1}^{k}=\sum_{k}\left(\partial x_{j} / \partial p_{1}\right) \partial z^{k} / \partial p_{1}$ is the indirect effect of $p_{1}$ on $x_{j}$ through the induced changes in the $z^{\prime} s$. Of course, in $(11 b)$ and $(11 c) \sigma_{v}^{k 1}=\sigma_{v}^{k m}=0$ by the definition of fixed factors, and only the indirect effect remains.

To evaluate the $\sigma_{\ell}^{\prime s}$ empirically, one requires the derivatives of the restricted cost function $F(\cdot)$ and of the long run demands for the fixed factors $\mathrm{H}^{\mathrm{k}}(\cdot)$ which appear in $(11 \mathrm{a}-11 \mathrm{c})$. The latter can be expressed in terms of the derivatives of $F(\cdot)$ by performing comparative statics on the system of envelope conditions given in (4) (see Brown and Christensen 1981 for details). It is important to emphasize that the derivation of $(11 a-11 c)$ uses the envelope conditions, and hence these formulas are valid only for $z=z^{*}$. This point also applies. to the long run cost elasticity, as we noted. There are two approaches to this problem. The first is to estimate the $z^{*}$ as those values which solve the system of envelope conditions (4), given the data on $y, p$ and $r$ and the parameter estimates of the restricted cost function, and then to evaluate $(11 a-11 c)$ at the estimated $z^{*}$ (Brown and Christensen 1981). One limitation of this approach is that it is not possible to determine whether the divergence between the estimated $z$ and the observed $z$ is statistically significant or simply reflects sampling error in the estimated $z$. An alternative approach which we propose is to test the hypothesis that $z=z^{*}$. If the hypothesis is not rejected, then $(11 a-11 c)$ can be evaluated at the observed levels of $z$. If the hypothesis is refected, the evidence supports a significant divergence between $z$ and $z$ and the first approach must be used. In the next section we develop such a test. 


\section{A Test of Static Equilibrium}

The test is conducted under the null hypothesis $\left(\mathrm{H}_{0}\right)$ that the static equilibrium levels of fixed factors are held. Under $\mathrm{H}_{0}$ the fully spectfled model consists of the restricted cost function, the conditional demands for the variable inputs, and the long run demands for fixed factors. That is, we have the following system of (structurally recursive) simultaneous equations :

$$
\begin{aligned}
v c & =F\left(y, p, z ; \beta_{0}\right)+\varepsilon, \\
x & =F_{p}\left(y, p, z ; \beta_{1}\right)+\varepsilon_{2} \\
z & =H\left(y, p, r ; \beta_{3}\right)+\varepsilon_{3}
\end{aligned}
$$

where the $\varepsilon^{\prime}$ 's are stochastic disturbances which reflect random optimization and other errors and which in general are correlated across equations, and the $\beta^{\prime}$ 's are parameter vectors. The validity of the restricted equilibrium framework (for arbitrary $z$ ) is a maintained hypothesis. This implies that $\beta_{1} \subset \beta_{0}$, that is the vector $\beta_{1}$ which parametrizes $(12 b)$ is a subset of $\beta_{0}$ in (12a). These cross-equation parameter constraints are maintained here. ${ }^{4}$ Also note that $\mathrm{H}_{0}$ allows for random error in the determination of the static equilibrium levels of $z$. This seems distinctly preferable to the more restrictive and less realistic hypothesis that $z=z^{*}$ exactly. It will turn out that the test of $\mathrm{H}_{0}$ which we propose is equivalent to the Hausman test for misspecification applied to the system of simultaneous equations $(12 a-12 c)$ (Hausman 1978). Since this is by no means obvious, we now discuss the intuition behind the test and then present it formally. 
The substantive implication of $\mathrm{H}_{0}$ is that $B_{2} C B_{0}$, since (12c) is derived from (12a) by using the envelope conditions. Partition the vector $B_{0}$ \&s $B_{0}=\left(B_{0}^{0}, B_{2}^{0}\right)$ where the elements of $B_{0}^{0}$ appear in (12a) but not in (12c) under $\mathrm{H}_{0}$. Then $\mathrm{H}_{0}$ implies the restrictions $B_{2}=B_{2}^{0}$. The most direct test of $\mathrm{H}_{0}$ is a standard likelihood ratio test of $B_{2}=B_{2}^{0}$. Let $\hat{B}$ be the asymptotically efficient, constrained estimator of $\beta$ from $(12 a-12 c)$ under the restrictions $B_{2}=B_{2}^{0}$ and $\tilde{B}$ be the unconstrained estimator from $(12 a-12 c)$, where both $\hat{B}$ and $\tilde{B}$ take account of the cross-equation correlation among the stochastic disturbances. The standard test is based on a comparison of the values of the likelihood function under $\hat{B}$ and $\tilde{B}$. In order for this test to be valid, $\hat{B}$ must be a consistent estimator of $B$ under $\mathrm{H}_{0}$ but inconsistent under $\mathrm{H}_{1}$, while $\tilde{B}$ must be consistent under both $\mathrm{H}_{0}$ and $\mathrm{H}_{1}$. This requirement is met only under a rather narrow class of alternative hypotheses, in particular that under $\mathrm{H}_{1}$ the observed $z$ are some arbitrary function of only $y, p$ and $r$. In this case (12c) remains properly specified under $H_{1}$, but with $B_{2} \neq B_{2}^{0}$. This specification of $H_{1}$ is too narrow, however, because almost any dynamic specification of the determination of $z$ will yield demand functions for $z$ which depend on additional variables beside $y, p$ and $r$. Under a broader formulation of $\mathrm{H}_{1}$, however, the standard test breaks down because then $(12 \mathrm{c}$ ) is misspecified under $\mathrm{H}_{1}$ and the unconstrained estimator is inconsistent. The inconsistency arises because the misspecification in (12c) is transmitted to $\tilde{B}$ through the covariance in the $E^{\prime} s$ used to construct $\tilde{B}$. A valid test can be developed by recognizing that, regardless of how $z$ is determined under $\mathrm{H}_{1}$, the system $(12 a-12 b)$ remains properly specified since $1 t$ holds for arbitrary $z$. Hence, we propose to test $H_{0}$ by comparing 
the asymptotically efficient, constrained estimator from $(12 a-12 c)$ under the restrictions $\beta_{2}-\beta_{2}^{0}, \hat{\beta}$, to another estimator obtained from $(12 a-12 b)$, $\tilde{B}$. As required for a valid test, $\hat{B}$ is consistent under $H_{0}$ but not under (the more general) $H_{1}$, while $\tilde{B}$ is a consistent estimator under both $H_{0}$ and $\mathrm{H}_{1}$.

The test procedure can be formalized as follows. Suppose that $(12 a-12 c)$ can be expressed as linear equations in the parameters. Write $(12 a-12 b)$ in stacked matrix form:

$$
\mathrm{Y}=\mathrm{X} \beta+\mathrm{U}
$$

where $Y$ is an $(m+1) T \times 1$ vector consisting of $T$ observations on variable cost followed by those on the $m$ variable factors, $X$ is an $(m+1) T \times k$ matrix on $y, p$ and $z$ ( $k$ in number), $B$ is $a(k \times 1)$ parameter vector, and $\mathrm{U}$ is an $(\mathrm{m}+1) \mathrm{T} \times 1$ vector of disturbances with $\mathrm{E}(\mathrm{U})=0$ and $E\left(U U^{\prime}\right)=I \otimes \Phi$ where $\mathrm{I}$ is a $\mathrm{T} \times \mathrm{T}$ identity matrix. Let the system augmented by (12c) be $\left[\begin{array}{l}\mathrm{Y} \\ \mathrm{Z}\end{array}\right]=\left[\begin{array}{l}\mathrm{X} \\ \mathrm{X}_{\mathrm{Z}}\end{array}\right] B+\left[\begin{array}{c}\mathrm{U} \\ \\ \varepsilon\end{array}\right]$

or

$$
\underline{Y}=\underline{X} \beta+\zeta
$$

where $z$ is an $n T \times 1$ vector of observations on the $n$ fixed factors, $x_{z}$ is an $n T \times k$ matrix on $y, p$ and $r$, and $\varepsilon$ is an $n T \times 1$ vector of disturbances with $E(\varepsilon)=0, E\left(\varepsilon \varepsilon^{\prime}\right)=I \otimes \Omega$, and $E\left(\varepsilon U^{\prime}\right)=I \otimes \psi$. Finally, let $E\left(\zeta \zeta^{\prime}\right)=$ I $8 \Sigma$, where $\Sigma=\left[\begin{array}{ll}0 & \psi \\ \psi & \Omega\end{array}\right]$. 
Since $z$ is endogenous under $H_{0}$ and appears in $X$ and $\underline{X}$, instrumental variable (IV) estimators are required. Let $P$ and $\underline{P}$ be conformable matrices of suitable instruments for $X$ and $X$ (such that $P^{\prime}\left(I \theta \Phi^{-1}\right) X$ and $\underline{P}^{\prime}\left(I \otimes \Sigma^{-1}\right) \underline{X}$ are square and nonsingular $)$. The generalized covariance IV estimators of $B$ from (13) and (14) are

$$
\begin{aligned}
\tilde{B} & =\left[P^{\prime}\left(I \otimes \Phi^{-1}\right) X\right]^{-1} P^{\prime}\left(I \otimes \Phi^{-1}\right) Y \\
& =B+\left[P^{\prime}\left(I \otimes \Sigma^{-1}\right) X\right]^{-1} P^{\prime}\left(I \otimes \Phi^{-1}\right) U
\end{aligned}
$$

and

$$
\begin{aligned}
\hat{B} & =\left[\underline{P^{\prime}}\left(I \otimes \Sigma^{-1}\right) \underline{X}\right]^{-1} P^{\prime}\left(I \otimes \Sigma^{-1}\right) \underline{Y} \\
& =B+\left[\underline{P}^{\prime}\left(I \otimes \Sigma^{-1}\right) \underline{X}\right]^{-1} \underline{P}^{\prime}\left(I \otimes \Sigma^{-1}\right) \zeta
\end{aligned}
$$

It follows that $\sqrt{\mathrm{T}}(\hat{\mathrm{B}}-B) \stackrel{\mathrm{A}}{\sim} \mathrm{N}\left(0, \mathrm{~V}_{0}\right)$ and $\sqrt{\mathrm{T}}(\tilde{B}-B) \stackrel{\mathrm{A}}{\sim} \mathrm{N}\left(0, \mathrm{~V}_{1}\right)$ where $\stackrel{A}{\sim}$ means asymptotically distributed, $v_{0}=\operatorname{plim}\left[\underline{P}^{\prime}\left(I \otimes \Sigma^{-1}\right) \underline{X} / T\right]^{-1}$ and $v_{1}=P \lim \left[P^{\prime}\left(I \otimes \Phi^{-1}\right) X / T\right]^{-1}$. From $(15)$ and (16)

$$
\begin{aligned}
\tilde{B}-\hat{B}= & {\left[P^{\prime}\left(I \otimes \Phi^{-1}\right) X\right]^{-1} P^{\prime}\left(I \otimes \Phi^{-1}\right) U } \\
& -\left[\underline{P}^{\prime}\left(I \otimes \Sigma^{-1}\right) \underline{X}\right]^{-1} \underline{P}^{\prime}\left(I \otimes \Sigma^{-1}\right) \zeta .
\end{aligned}
$$

Hence, $\sqrt{T}(\bar{B}-\hat{B}) \stackrel{A}{\sim} N(0, V)$ where it can be shown that $V=v_{1}-v_{0} \cdot 5$ Let $\hat{\mathrm{V}}$ be any consistent estimator of $\mathrm{V}$. Then we obtain the test statistic $M=T(\tilde{B}-\hat{B}) \cdot \hat{V}^{-1}(\tilde{B}-\hat{B}) \stackrel{A}{\sim} x_{q}^{2}$ where $q$ is the number of restrictions being tested (i.e., embodied in $B_{2}=B_{2}^{0}$ ).

To test the null hypothesis that all fixed factors are at their static equilibrium levels, the computed test statistic $M$ is compared to the critical value of the Chi-square deviate with $q$ degrees of freedom. 
The procedure can also be used to test the hypothesis that only a subset of fixed Inputs are at their optimal levels, leaving the others free to be at arbitrary levels. In this case the estimator $\bar{\beta}$ is defined as before, but $\hat{B}$ is obtained from $(12 a-12 b)$ plus the subset of (12c) corresponding to the fixed factors to be tested. Finally, it may be recognized that the test procedure provided here is equivalent to a Hausman test for specification error in a system of simultaneous equations (Hausman 1978). Hence, the existing literature on the power of the Hausman test is relevant here (e.8., Hausman 1978; Holly 1982).

\section{Rate of Return to Quasi-Fixed Factors}

In this section we show how to derive estimates of the Internal rates of return (IRR) to fixed factors from the restricted equilibrium framework. One preliminary point is in order. As noted in Section 1, the restricted cost function must be convex in $z$, and one of the implications of this condition is that the long run demand functions for $z$ are downward sloping (i.e., the shadow price $-F_{z}$ declines with $z$ ). Recall that the static equilibrium level of $z, z^{*}$, is determined by equalizing the shadow price and the opportunity cost of funds. Since (as we show below) the shadow price is directly related to the IRR, it follows that a divergence between the IRR at the observed $z$ and the opportunity cost of funds is a reflection of a divergence between the observed and static equilibrium levels of $z$. In fact the two problems can be viewed as the duals of each other. As a consequence it is meaningful to estimate the IRR only if a statistically significant divergence between $z$ and $z^{*}$ has been established. Therefore, the test 
of static equilibrium proposed in Section 2 should be conducted prior to the computation of the IRR to fixed factors.

In the present framework investments in 2 shift the restricted (or variable) cost curve downward and establish a new long run equilibrium price for output. The market price, however, may adjust with some lag. In each period the gross private returns to the investment ave the difference between total revenue and the new level of short run costs, evaluated at the prevalling short run equilibrium. These rents arise from the firm's temporary monopoly power resulting from the cost reduction or, equivalently, the sluggishness of price adjustment. The size of these rents depends both on the level of the new equilibrium price and the speed of adjustment toward it, and hence varies with the specific market structure under consideration. In this section and in the subsequent empirical application we analyze the case of a regulated, single product monopoly. (The modification for an unregulated competitive firm will be noted later.) No assumption is made regarding the degree of returns to scale. The firm is constrained to earn zero economic profits eventually. That is, the regulatory authorities fix the price of output at the intersection of the demand and average total cost curves, possibly with some lag, and the firm is required to meet demand at that price. The measure of average total cost used by the regulators for price determination includes a remuneration to the surviving (1.e., undepreciated) stock of fixed inputs. While in practice the determination of this "allowed return" is a complicated procedure, we assume that it reflects the opportunity cost of funds, or the market rental price of the fixed factors. 
Suppose the firm makes an investment in $z$ which lowers both the average variable and average total cost curves. If there is no regulatory lag, the price of output adjust immediately to the new level of average total cost and the Investment earns only the market rental price which 1s reflected in that cost. However, if there is any regulatory lag in adjusting the price, the firm also enfoys some rents and the total private return to the investment exceeds its opportunity cost. ${ }^{6}$ other things equal, the realized private returns vary directly with the length of the regulatory lag. Since static equilibrium requires that the marginal investment in $z$ earn only the normal rate of return, the question which arises is whether (and under what condition) there can be an equilibrium level of $z$ in the presence of regulatory lag. This can occur only if the marginal investment in $z$ does not shift the total cost curve since in this case the equilibrium price of output remains unchanged, there are no realized rents to the investment and the effect of regulatory lag is thereby neutralized. This condition holds if and only if the reduction in variable costs just equals the market rental price of $z$, which is exactly the familiar envelope condition (equation (4) above). We conclude that in this regulated environment with sluggish price adjustment the static equilfbrium level of $z, z^{*}$, is defined by the standard envelope condition. This conclusion does not imply that $z^{*}$ is independent of regulatory lag. The length of regulatory lag affects $z$ through its impact on the rents which accrue to investment in $z$. For example, a slower price adjustment generates larger private returns to such investment and hence a larger equilibrium stock of fixed factors $z$. 
We have argued that the private benefits from an investment in the stock of fixed input $z$ consists of a stream of rents over time due to regulatory 1 ag in adjusting the output price, plus the market rental price paid each period to the surviving stock $z$. The rents which accrue each period are the difference between the prevalling output price and the new level of average total cost, multiplied by the level of output. The time path of private returns is determined by three parameters. The first is the rate of growth of demand for output. Given the exogenously set price, the level of output and hence the size of the rents depend on the level of demand. The second is the rate of price adjustment toward the new, lower level of average cost. The third factor is somewhat more subtle. A unit of investment today raises by one unit the current stock of $z$. This stock represents the productive capacity of the cumulated past investments in $z$ and it is this "surviving" stock which appears in the restricted cost function. In general there will be some decay in the productive capacity of an investment as it ages, which we refer to as the rate of deterioration. Therefore, an investment today raises the surviving stock of $z$ by a diminishing amount over time, or put another way, it reduces the average total cost by an amount which erodes over time as deterioration sets in. This rate of deterioration affects the private returns in two ways. First, it shrinks the realized rents which accrue to the initial investment. Second, it reduces the effective remuneration for the opportunity cost of funds, since that compensation is paid on the basis of the surviving stock of $z$.

On the basis of the preceding discussion we can formalize the derivation of the IRR as follows. Define the average total cost function 


$$
\bar{C}=\bar{F}(y, p, z)+r \bar{z} \cdot
$$

where a bar denotes normalization by the level of output, $F(\cdot)$ is the restricted cost function, and $r$ is a vector of market rental prices for z. Note that $\partial \bar{c} / \partial z$. may be positive or negative and it will equal zero only if the envelope conditions hold (see Section 1).

Consider a unit investment in a particular quasi-fixed factor $z$ (we omit the identifying subscript for simplicity). The marginal net (internal) rate of return to this investment at time zero, $\rho$, is defined by the equation

$$
e^{\theta \rho}=\int_{0}^{\infty} B_{t} e^{-\rho t} d t
$$

where $\theta$ is the gestation 1 ag between the investment and its impact on $F(\cdot)$ (assumed to be a fixed rather than a distributed lag, for simplicity), and $B_{t}$ denotes the gross private returns at time $t$ from the investment. Assume that the demand for output grows at rate $g$. Let $\delta$ be the rate of adjustment of the output price toward the new level of average cost and $\phi$ be the rate of deterioration. Then

$$
B_{t}=\left(P_{t}-\bar{C}_{t}\right) y_{0} e^{g t}+r e^{-\phi t}
$$

where the first term represents the rents accruing at time $t$ and the second is the normal remuneration to the surviving piece of the investment. To capture the price adjustment let $\mathrm{p}_{t}-\overline{\mathrm{C}}_{t}=\left(\overline{\mathrm{C}}_{0}-\overline{\mathrm{C}}_{\mathrm{t}}\right) \mathrm{e}^{-\delta \mathrm{t}}$. But by definition $\overline{\mathrm{C}}_{t}=\overline{\mathrm{C}}_{0}+\partial \overline{\mathrm{C}}_{0} / \partial \mathrm{z}_{t}$, and from the argument about deterioration $\partial \overline{\mathrm{C}}_{0} / \partial z_{t}=\left(\partial \overline{\mathrm{C}}_{0} / \partial z_{0}\right) \mathrm{e}^{-\phi t}$. Substituting these relations into $(20)$ and noting $\mathrm{C}_{0}=\overline{\mathrm{C}}_{0} \mathrm{y}_{0}$, we obtain 
(21)

$$
B_{t}=-\left(\partial C_{0} / \partial z_{0}\right) e^{(g-\delta-\phi) t}+r e^{-\phi t}
$$

Using (18) and (21) In (19) and performing the integration (assuming $\delta+\phi>8)$, we can write the IRR, $\rho$, in the following implicit form:

$$
e^{\theta \rho}(\rho+\delta+\phi-8)-r\left(\frac{\delta-g}{\rho+\phi}\right)=-\frac{\partial F_{0}}{\partial z_{0}}
$$

We know from the earlier discussion that when the envelope condition holds the investment earns only the opportunity cost of funds. Then the net rate of return is equal to the (appropriate) market rate of interest, 1 . In other words, $p=1$ when $\partial C_{0} / \partial z_{0}=0$. Using this condition and (18) in (22), we can solve for the market rental price, $r$. This yields :

$$
\mathbf{r}=\mathbf{e}^{\theta 1}(i+\phi)
$$

Equation (23) defines the appropriate market rental price for $z$ to be used in the empirical work when the envelope conditions are imposed (neglecting tax parameters). Substituting it back into (22) we obtain

$$
e^{\theta p}(p+\delta+\phi-8)-e^{\theta i}(\delta-g)\left(\frac{i+\phi}{\rho+\phi}\right)=-\frac{\partial F_{0}}{\partial z_{0}} .
$$

Equation (24) is the nonlinear equation which implicitly defines the IRR, $\rho$, given the values for the other parameters. The term $-\partial \mathrm{F}_{0} / \partial \mathrm{z}_{0}$ represents the shadow price of $z$, evaluated at the observed (not the static equilibrium) level of $z$. It is retrieved from the estimated restricted cost function (1.e., using the system of equations (12a-12b) in Section 2). 
The discussion to this point has been based on a regulated firm. The derivation of the IRR for an unregulated competitive firm can be viewed as a spectal case, If we are willing to maintain the assumption that the level of output is exogenous to the firm. (Otherwise a decision rule for the determination of output must be incorporated into the analysis.) For the competitive firm the only source of private returns to investments in $z$ are the rents which arise from sluggish price adjustment in the market. The second set of terms in (24) vanishes and the equation for the IRR collapses to $e^{\theta \delta}(\rho+\delta+\phi-g)=-\partial F_{0} / \partial z_{0}$. The term $\delta+\phi-g$ can be interpreted as the rate of obsolescence of the value of the fixed asset, reflecting price adjustment $\delta$, deterioration $\phi$, and the capital gains due to market growth, 8 . An expression of this form can be found in Pakes and Schankerman (1984). 7

As noted, the computation of the IRR requires an estimate of the shadow price, $-\partial \mathrm{F}_{0} / \partial z_{0}$, which can be obtained from the estimated restricted cost function. This shadow price may also be useful in studying the growth in total factor productivity of firms or industries in which static equilibrium is violated. In an important paper Berndt and Fuss (1982) analyze the nonparametric measurement of productivity In cases where fixed factors diverge from their static equilibrium levels. They show that measures based on the market rental prices of fixed factors confound under- or overutilization of those factors with true productivity growth. They prove that the correct remedy is to use the (ex ante) shadow prices in place of market rental prices for the fixed inputs, and they suggest the use of Tobin's - q to approximate the expected shadow price. Our framework delivers direct estimates of the shadow prices 
(though ex post ones) " which could also be used to implement the BerndtFuss proposal. 8 This may be a particularly attractive alternative in models with more than one fixed factor, since it is not clear to us how Tobin's-q can be used to identify the shadow prices of several fixed inputs simultaneously.

\section{An Empirical Application}

In this section we provide an empirical application of the techniques developed in Sections 1-3 to data on the Bell System for the period 1947-1976. We first briefly summarize the emplrical framework and then present and discuss the results.

The empirical work is based on a generalized Cobb-Douglas restricted cost function. Letting lower case letters denote loragithms; the form we use is

$$
v c-y=\alpha_{0}+\sum_{1} \alpha_{i} p_{1}+\sum_{k} \beta_{k} z_{k}+\sum_{k \neq l} \sum_{\ell} \gamma_{k l} z_{k} z_{l}+\sum_{1} \sum_{k} \lambda_{1 k} p_{1} z_{k}
$$

where $1, j=1, \ldots$, m denote the variable inputs, $k, l=1, \ldots, n$ denote fixed factors, and all variables are defined around some expansion point. The form in (25) is a special case of the more general translog specification, allowing only linear interactions among variable factor prices and fixed inputs and imposing constant short run average cost. The model we estimate is based on two variable inputs, labor and materials, and two fixed inputs, the stocks of capital and of R\&D. We experimented with the full translog specification and found that it was seriously overparametrized. The time series data used here cannot sustain such complicated parametric specifications. ${ }^{9}$ Christensen, Cummings and 
Schoech (1980) used simflar data and encountered the same difficulties in a model with one fixed input, and the presence of two fixed factors here only exacerbates the problem.

Applying Shephard's Lemma to (25) yields the set of share equations for variable inputs

$$
s_{1}=\alpha_{1}+\sum_{k} \lambda_{1 k^{2} k}
$$

where $S_{1}=P_{1} X_{1} / V C$ is the share of input 1 in variable cost. If in addition the fixed factors are at their static equilibrium levels, the envelope conditions (4) hold. Applying (4) to (25) yields the equations

$$
-\pi_{k}=\beta_{k}+\sum_{\ell \neq k} \gamma_{k \ell} z_{l}+\sum \lambda_{1 k} p_{1}
$$

where $\pi_{k}=r_{k} z_{k} / V C$ is the ratio of the fixed cost for input $k$ to variable cost. Equations (27) hold only at the static equilibrium levels of $z_{k}$. We impose the theoretical requirement of linear homogeneity in input prices using the restrictions $\sum_{1} \alpha_{1}=1$ and $\underset{1}{\sum_{1 k}} \lambda_{1 k}=0$. The symetry restriction on $\lambda_{i k}$ and $\gamma_{k \ell}$ are also imposed. The formulation in (25) imposes a unitary variable cost elasticity and elasticity of substitution (AES) between variable inputs. The long run cost elasticity is given by $n_{\ell}=\left(1+\pi^{*}\right)^{-1}$ where $\pi^{*}=\sum_{k} \pi_{k}^{*}$ and the $\pi_{k}^{*}$ 's are evaluated at the static equilibrium levels of all variables. The long run AES are retrieved using the procedure outlined in Section 1.10

The bastc model consists of (25) and (26). Thts corresponds to $(12 a-12 b)$ in Section 3 and makes no assumption about how fixed factors 
are determined. To conduct the tests of static equilibrium, we also require that the envelope conditions (27) for the fixed factors being tested be included. We specify jointly normal, additive disturbances with contemporaneous correlation across equations. The equations are estimated by 1terated three stage least squares (3SLS), using a set of instruments for the fixed factors. ${ }^{11}$ One vartable share equation is dropped due to the normalization on shares, but the parameter estimates are Invartant to which equation ts omfted. A diagonal spectfication for first order autocorrelation 18 made in accordance with the requirements of a singular system of equations (Berndt and Savin 1975).

We use annual data for the Bell System over the perfod 1947-1976. The data are described in detall In Nadirf and Schankerman (1981a) and only a brief summary is provided here. ${ }^{12}$ The measure of aggregate output Is the sum of operating revenues for four service categories--local service, Intrastate toll, interstate toll, and a small miscellaneous category. The operating revenues for each category are deflated by a category-spectfic Paasche price index. The quantity of labor input is the man hours actually worked adjusted for changes in the composition of the work force. Man hours are classlfled into twenty-two categories based on occupation and years of service and a composition adjustment is made by welghting the man hours in each category by the relative wage rates in the base year, 1967. (The data were not avallable to use varying relative wage rates as weights.) An fmplictt price index for labor is constructed as the ratio of total employee compensation to the quantity of labor Input. The materials Input consists of stx categories of materfals, rents and supplies, each separately deflated. An implicit 
price index for the materials input is then constructed.

The stock of capital is the sum of net tangible plant, cash, net accounts receivable and inventories. Tangible plant, which accounts for the bulk of capital stock, is constructed from twenty-three categories of capital, each identifled by vintage, separately depreclated and deflated using the Bell Telephone System Plant (Laspeyres) Indices. As required by equation (23), the service price of capital is constructed as the sum of the cost of investment funds and the rate of depreciation, multiplied by an investment goods deflator and adjusted for various tax parameters in the standard way. 13

The stock of R\&D is constructed as a geometrically weighted sum of deflated non-military R\&D expenditures by the Bell System, lagged for years. The $1 a g$ is designed to reflect the mean gestation period between R\&D expenditure and its impact on the level of variable costs. R\&D flows are deflated by the implicit GNP price index. The cumulation of R\&D flows is based on an assumed rate of deterioration of 0.05 (but the empirical results are robust to alternative assumptions). The service price of $R \& D$ is constructed as required by equation (23), multiplied by the implicit GNP deflator. No adjustment for tax parameters is made because R\&D expenditures are treated for tax purposes by the company as an operating expense rather than as a capital asset.

Table 1 presents the parameter estimates for the basic model. As required by the theory, increases in the stocks of fixed factors reduce the level of variable cost $\left(\beta_{k}<0, \beta_{r}<0\right)$. The estimated restricted cost function satisfies the theoretical requirements of monotonicity and concavity in variable factor prices, and convexity in fixed factors, at 
every sample observation. The fits of the share equations are rather low, but this is not surprising since these equations have been almost firstdifferenced in the estimation $(\rho-0.9)$.

Before turning to the Implied characterization of the long run cost function, we first must conduct the tests of static equilibrium for the fixed factors using the procedure developed in section 2 . The test is based on a comparison between the parameter estimates from (25) and (26), and the estimates from (25), (26) and the subset of (27) which corresponds to the fixed factors being tested. The procedure allows us to test capital and R\&D separately (leaving the other free) as well as jointly. Table 2 summarizes the results. The first row indicates strong rejection of static equilibrium for capital. The computed test statistic of 17.8 greatly exceeds the critical value of 7.8 at the five percent level of significance. By contrast, the second row shows that we cannot reject the hypothesis of static equilibrium for R\&D when it is tested separately. However, the joint hypothesis that both capital and R\&D are at their static equilibrium values during the sample period is easily rejected. There is of course no contradiction between these results for the Individual and joint hypothesis tests. It is worth noting, however, that the nonrejection of static equilibrium for R\&D is not robust. If we specialize the restricted cost function in (25) to a Cobb Douglas form and rerun the tests, we find that static equilibrium is rejected for both R\&D and capital, Individually and jolntly.

We can use the parameter estimates in Table 1 to solve for the Implied static equilibrium levels of capital and R\&D, and then use these values to retrieve characteristics of the long run cost function. Table 3 
Table 1. Parameter Estimates for the Restricted Cost Function

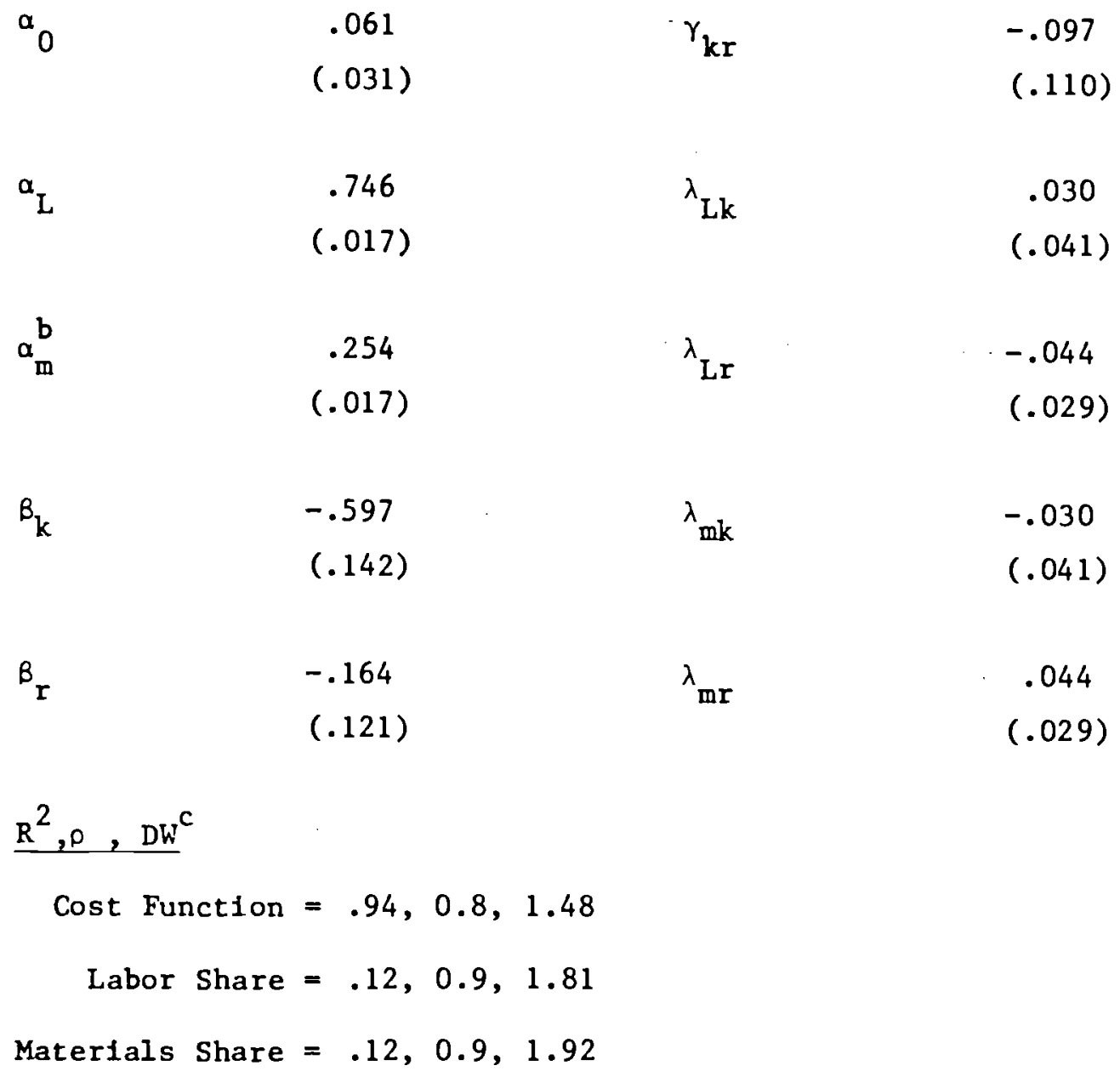

Notes

a Estimated standard errors are in parentheses.

$b$ Note that $\alpha_{m}=1-\alpha_{L}, \lambda_{m k}=-\lambda_{L k}$ and $\lambda_{m r}=-\lambda_{L r}$.

c $\rho$ refers to the autocorrelation coefficient used to adjust the equation. 
Table 2. Tests of Static Equilibrium for Fixed Factors

\begin{tabular}{lccc} 
Quasi-Fixed Factor & $\mathrm{M}^{\mathrm{a}}$ & d.f. & Critical $\mathrm{X}^{2} .05$ \\
\hline Capital & 17.8 & 3 & 7.8 \\
R\&D & 6.1 & 3 & 7.8 \\
Capital and R\&D & 32.9 & 6 & 12.6 \\
\hline
\end{tabular}

a The test statistic $M$ (described in Section 2) distributes asymptotically as a Chi-square deviate. 
Table 3. Characteristics of the Long Run Cost Function ${ }^{\text {a }}$

$\begin{array}{lccc}\sigma_{L K} & 1.07 & \varepsilon_{L L} & -0.69 \\ \sigma_{L M} & 1.10 & \varepsilon_{M M} & -0.88 \\ \sigma_{L R} & 2.14 & \varepsilon_{K K} & -0.74 \\ \sigma_{M K} & 1.38 & \varepsilon_{R R} & -0.67 \\ \sigma_{M R} & -0.55,0.13 & n_{\ell} & 0.55 \\ \sigma_{K R} & -0.48,0.28 & & \end{array}$

a The terms $\sigma, \varepsilon$ and $n_{\ell}$ refer to the AES, own elasticitles of factor demand, and cost elasticity, respectively. Reported values are those at the expansion point of the sample (1961) but they are stable over time--except for $\sigma_{\mathrm{MR}}$ and $\sigma_{\mathrm{KR}}$. These estimates are trended; the first reported number is the average over the perlod 1948-1961 and the second for 19621976. 
presents the results. The AES Indicate considerable long run substitution among inputs, except between $R \& D$ and materials and $R \& D$ and capital where the estimates vary over time and are much smaller. The long run elasticities of factor demand are negative, as required by theory. It is interesting to note that the factor demands are price inelastic and similar across inputs. The long run cost elasticity is estimated at 0.55 , which implies a scale elasticity of about 1.8. This is almost identical to the finding In Nadiri and Schankerman (1981) but at the upper end of the range reported in Christensen, Cummings and Schoech (1980). 14

Table 4 summartzes information on the divergence between observed levels of capital and R\&D and the static equilibrium levels implied by the estimated restricted cost function. The first two rows give the ratio of observed to static equilibrium stocks of capital and R\&D by subperiods. The evidence suggests that the Bell System was substantially overcapitalized, by between 21 and 50 percent depending on the subperiod. The extent of overcapitalization increased sharply during the decade 1958-1967, which interestingly is the period when the Bell System undertook large investment programs in new technologies. This finding of overcapitalization is consistent with the well-known Averch-Johnson effect of rate of return regulation, for which there is some evidence in the literature (Courville 1974, Cowing 1978, and Spann 1974 on the electric utility industry). Other interpretations are suggested later. In sharp contrast, Table 4 indicates substantial underinvestment in $R \& D$. The static equilibrium stock of $R \& D$ is about three times as large as the observed one. This result may not be as unreasonable as it first appears. The average observed $R \& D$ intensity (R\&D expenditure/total cost) for the Bell System during 1948-1976 was 
Table 4. Characteristics of the Divergence Between Observed and Static Equilibrium Levels of Capital and R\&D

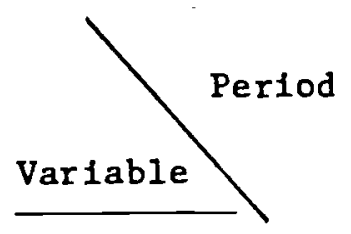

$$
\text { 1948-1957 }
$$

$1958-1967$

$1968-1973$

$\mathrm{K} / \mathrm{K}^{*}$

1.21

1.47

1.50

$$
\mathrm{R} / \mathrm{R}^{*}
$$

0.49

0.32

0.32

$-\mathrm{F}_{\mathrm{K}} / \mathrm{x}_{\mathrm{K}}$

0.68

0.51

0.56

$-F_{R} / r_{R}$

3.19

3.50

3.69

$\left(c-c^{*}\right) / C^{*}$

0.048

0.073

0.117 
only 2.1 percent, which is less than half of the R\&D intensity in other technologically dynamic Industries.such as Chemicals and Professional and Scientific Instruments (National Science Foundation, annual). The static equilibrium R\&D intensity which we compute is about 7.3 percent, which may be plausible considering the scientific richness of the telecommunications field. Of course, in order to make a complete and valid comparison other factors (such as the degree of vertical integration) must be taken Into account.

The third and fourth rows in Table 4 compare the shadow and market rental prices for capital and R\&D. As noted in Section 3 , the differences between shadow and market rental prices are the dual of the divergences between observed and static equilibrium stocks of these assets. Following Berndt and Fuss (1982), the shadow prices should be used for the measurement of total factor productivity. All of the avallable measurements of productivity growth in the Bell System utilize market rental prices, but the evidence in Table 4 suggests that this might yield quite inaccurate results. Finally, Table 4 provides the percentage deviation of actual total costs from the level which would occur if all factors were at their static equilibrium levels. The latter is evaluated by using equilibrium stocks of capital and R\&D, and the associated equilibrium levels of variable factors which depend on those stocks. The results indicate that "inoptimal" choice of capital and R\&D raised total costs by about five to ten percent over the sample period.

One important qualification is in order. The preceding evidence does not imply that the Bell System's use of capital and R\&D was inoptimal given the set of constraints it actually faced, but rather than the static 
equilibrium levels were not held. - The cholre may well have been optimal under a richer set of constraints such as rate of return regulation or severe costs of adjustment. The only conclusion we draw at this stage Is that a static equilibrium representation of the Bell System is Inadequate.

The remaining task is to compute the net internal rates of return to cap1tal and $R \& D$. These rates of return, $\rho$, are computed as the solution to the nonlinear equation (see Section 3):

$$
e^{\theta \rho}(\rho+\delta+\phi-g)-e^{\theta 1}(\delta-g)\left(\frac{1+\phi}{\rho+\phi}\right)+\frac{\partial F}{\partial z}=0
$$

whene $\theta$ is the gestation lag, 8 is the expected rate of growth of output, 1 is the opportunity cost of funds, $\phi$ the rate of deterioration, $\delta$ is the rate of output price adjustment, and $\partial F / \partial z$ is the estimated shadow price of the asset (evaluated at the expansion point, 1961, but the results are robust). We use the following parameter values: $1=.055$, $g=.07, \phi=.05, \theta=0,2,4$ (years), and $\delta=.25, .30, .35 .15$ Since the opportunity cost of funds is measured net of taxes, the computed rates of return are after-tax.

Table 5 presents the results. The net rate of return to capital Is estimated at about 4.5 percent and is robust to variations in $\theta$ and $\delta$. The fact that this rate of return is lower than the measured opportunity cost of funds reflects the overcapitalization (relative to static equilibrium) found earlier. The net rate of return to R\&D is markedly higher, reflecting underinvestment in this input. The results are reasonably robust to variations in $\delta$ and $\theta$, except in the case where $\theta=0.16$ Pakes and Schankerman (1984) conclude that $\theta \simeq 2$ for 
Table 5. Net Rates of Return to Capital and R\&D in the Bell System

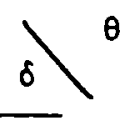

.25

.30

.35

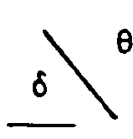

.25

.30

.35
Capital

0

2

4

.041

.041

.044

.044

.045

.046

.046

.047

.046

$\underline{R \& D}$

0

2

4

.242

.150

.115

.225

.138

.109

.198

.128

.102 
manufacturing industries and this probably understates the gestation lag for telecommuntcations research. Therefore, we conclude from Table 5 that the net rate of return to $R \& D$ in the Bell System is between 10 and 15 percent.

\section{Concluding Remarks}

In the literature two methodologies are used to estimate the structure of production and factor demand equations. One is the static equilibrium approach, which treats all factors of production as variable and assumes away the problem of costs of adjustment. The other are dynamic equilibrium models which are based on the theory of restricted cost functions and arbitrarily assign costs of adjustment to certain inputs. What is missing from the literature is an analytic approach to testing statistically which class of models is appropriate for the analysis of a given set of data. The main contribution of this paper is to provide a statistical test to assess the adequacy of the static equilibrium model, which should be used prior to specifying a fully dynamic equilibrium model. We also show how restricted cost function models can be exploited to investigate some important issues such as the calculation of the rates of return to fixed factors, the determination of over- or underinvestment in particular assets, and the distinction between short run excess capacity and long run economies of scale. The specific contributions of the paper may be summarized briefly. First, a restricted cost function framework is specified and it is shown how to derive expressions for the long run cost elasticity and elasticities of substitution. Second, a statistical test of static equilibrium is 
formulated by using the restricted equilibrium model in confunction with the envelope conditions which characterize static equilibrium for the fixed factors. Our test procedure is equivalent to a Hausman test for specification error in a system of simultaneous equations. Third, we provide a method of computing the internal rates of return to fixed factors and show how these rates of return depend on the shadow prices of fixed factors, the growth of demand for output, the rate of price adjustment, and the rate of deterforation of the stocks of flxed factors. We also show that a divergence between the rate of return (at the observed level of fixed factors) and the opportunity cost of funds is the dual of the divergence between the observed and static equilibrium levels of fixed factors. The methodology also provides direct estimates of the shadow price of flxed inputs which can be used to adjust measured productivity growth in cases where fixed factors diverge from their static equilibrium levels.

To 1llustrate our methodology we have used data for the Bell System for the period 1947-1976 to estimate a generalized Cobb-Douglas restricted cost function with capital and R\&D treated as fixed factors. The empirfcal results indicate substantial long run economies of scale and substitution among most of the inputs. The hypothesis of static equilibrium is rejected strongly for capital and (wlth qualification) for R\&D. The evidence indicates that the Bell System was substantially overcapttalized, particularly during 1958-1967 when it undertook large investment programs in new technologles. However, there is evidence of substantial underinvestment in R\&D for the Bell System. The results also suggest substantial divergence between the shadow and market rental 
prices for capital and R\&D,-which reflects-the divergence between the observed and optimal levels of fixed factors. The same picture emerges when the rates of return are considered. The net rates of return to capital and R\&D are 4.5 percent and 10-15 percent respectively, compared to an opportunity cost of funds of about 5.5 percent. The "inoptimal" choice of fixed factors raised total costs by five to ten percent over the static equilibrium level.

The approach proposed in this paper is applicable to other areas of economics and is not confined to empirical studies of production and cost functions. It provides a procedure for researchers to examine whether static equilibrium models are satisfactory or whether a fully dynamic equilibrium model is warranted, and it suggests a number of issues which can be explored prior to estimation of dynamic models. 


\section{Footnotes}

* We would like to thank R. Frydman, R. Gordon, P. Mohnen, and J. Ramsey for constructive comments on earlier versions of this paper. This research was supported by the National Science Foundation Grant PRS-7727048, and by a grant from the American Telephone and Telegraph Company.

1. The relevant literature is quite extensive. Leading examples include Morrison and Berndt (1981), Denny, Fuss and Waverman (1981), and Epstein and Denny (1983). For a partial review of the literature see Berndt, Morrison and Watkins (1981).

2. Restricted cost functions fall under the more general heading of restricted profit functions, of which they are a special case. See Lau (1976).

3. Caves, Christensen and Swanson (CCS) show that the degree of returns to scale, RTS, can be expressed as $\operatorname{RTS}=(1-\partial \ln V C / \partial \ln z) n_{v}^{-1}$. At $z=z^{*}$ this reduces to equation (8) (where RTS $=n_{\ell}^{-1}$ ), but in general it implies that the degree of long run scale economies can be retrieved from the restricted cost function at arbitrary levels of $z$. The equation derived by CCS is correct. The problem with their analysis is that RTS is not the correct measure of scale economies in general. The concept of RTS is based on equiproportional increases in all inputs, including $z$. As Hanoch (1975) showed, the correct concept of scale economies is $n_{l}^{-1}$, measured along the expansion path. The two concepts are the same only if the production structure is homothetic, in which case the expansion path is linear and equiproportional expansion of all inputs corresponds to 
long run cost minimizing behavior. That 1s, the envelope conditions are assumed 1mplicitly to hold, and $z=z^{\star}$. Therefore, the only case when RTS Is the correct measure of scale economies corresponds to the case where $z=z^{*}$. If the technology is not homothetic, the CCS measure of RTS is equivalent to the reciprocal of the short run cost elasticity. To show this, suppose that all inputs are increased equiproportionally but not along the expansion path. This is equivalent in (7) to assuming that $n_{z y}=n_{s}$, since if all inputs grow at the same rate then (short run) costs must also grow at that rate. Then (7) reduces to $n_{s}=\left(1+\pi+\pi_{d}\right)^{-1} n_{v}$. Since $\pi+\pi_{d}=-\partial \ln \mathrm{VC} / \partial \ln z, n_{s}=(1-\partial \ln \mathrm{VC} / \partial \ln z)^{-1} n_{v}$. Then the CCS result is simply $\operatorname{RTS}=n_{s}^{-1}$. In other words the cCS result should be interpreted as a spectal case of (7), pertaining to the short run cost elasticity. As indicated before, in order for 1 t to relate to long run scale economies, the assumption $z=z^{*}$ must be made.

4. The assumption of restricted cost minimizing behavior could be examined by testing these cross-equation parameter constraints. Appelbaum (1978) takes this approach in the context of an unrestricted cost function. 5. To prove this note that it follows from (17) that plim $T(\bar{B}-\hat{B}) \cdot$ $(\tilde{B}-\hat{B})^{\prime}=v_{0}+v_{1}-W-W^{\prime}$ where $W=v_{1} \operatorname{Plim}\left[P^{\prime}\left(I \otimes \Phi^{-1}\right) U \zeta T^{-1}\left(I \otimes \Sigma^{-1}\right) \underline{P}\right] v_{0}$. But $\operatorname{plim}(U \zeta / T)=(\phi \psi)$ and $\Sigma^{-1}=\left[\begin{array}{ll}\Phi & \psi^{\prime} \\ \Psi & \Omega\end{array}\right]^{-1}$. Using the formula for a partitloned inverse one obtalns $\operatorname{plim}(U \zeta / T)\left(I \otimes \Sigma^{-1}\right)=\left(\begin{array}{ll}I & 0\end{array}\right)$. Now partition $\underline{P}$ as $\underline{P}=\left(P_{z}\right)^{\prime}$ where $P_{z}$ contains instruments in $\underline{P}$ but not in $P$. Using these results in the expression for $\mathrm{W}$ yields $\mathrm{W}=\mathrm{v}_{1} \mathrm{v}_{1}^{-1} \mathrm{v}_{0}=\mathrm{v}_{0}$. Since $\mathrm{v}_{0}$ is symmetric we get $\operatorname{pllmT}(\tilde{B}-\hat{B})(\tilde{B}-\hat{B})^{\prime}=v_{0}-v_{1}-v_{0}-v_{0}^{\prime}=v_{1}-v_{0}$.

6. This assumes, as stated, that the marginal investment in $z$ 
lowers the (average) total cost-curve. This will hold if the stock of $\mathrm{z}$ Is less than the static equilibrium level (since from (3) $C_{z}=F_{z}+r$ and $-F_{z}>$ If $z<z^{*}$ ). If $z>z^{*}$ then the investment will raise average total costs even though it reduces average variable costs. Then regulatory lag induces negative rents and the private return falls short of the opportunity cost. The conclusion which follows, however, holds for both cases.

7. Letting $\delta^{*}=\delta+\phi-g$, note that the equation for the IRR is $e^{\theta \rho}\left(\rho+\delta^{*}\right)=-\partial F_{0} / \partial z_{0}$, while in equilibrium $e^{\theta 1}\left(1+\delta^{*}\right)=r$. This makes It clear that departures of the shadow price from the market rental price are reflections of the divergence between the IRR and the opportunity cost of funds.

8. An alternative but less direct approach, suggested by Nadiri and Schankerman (1981b), is to compute the IRR to the fixed factors and then use them (instead of the opportunity cost of funds) to evaluate the service prices of the flxed inputs. Since a divergence between the shadow and market rental prices reflects a deviation of the IRR from the cost of funds (see note 7), this approach is equivalent to the Berndt-Fuss proposal.

9. It is worth noting that the specification in (25) is marginally accepted at the 0.01 level when tested against the full translog form. The computed $x_{8}^{2}$ is 20.0 , against a critical value of 20.1 .

10. In the full translog specification, the long run AES take on a very simple form if there is only one fixed factor--which arises frequently in practice. Since this form does not utllize any of the translog parameters which are omitted in (25), we state the result here. 
The derivation is lengthy and is avallable on request. Using the notation in (25) the results are:

$$
\sigma^{1 j}=(1+\pi)\left[\sigma_{v}^{1 j}-\left(\psi_{1}-s_{1} \pi\right)\left(\psi_{j}-s_{j} \pi\right) / s_{i} s_{j} \pi\right]
$$

and $\sigma^{1 z}=\left(1-\Psi_{1} / S_{1} \pi\right)$, where all variables are evaluated at their static equilibrium values.

11. The instruments include the exogenous variables in the model plus a time trend and its square, the level of output and its square, and the stock of federal government capital. The empirical results without Instruments are not very different from those reported in the text.

12. The raw data used in this paper were provided by the Bell System. These data are proprietary and inquiries regarding them should be addressed to M. A. Chaudry at the American Telephone and Telegraph Company •

13. The service price of capital is constructed as $r=P_{I}(1-u z-w)(1+\phi) /(1-u)+\tau$ where $P_{I}$ is the investment goods deflator, $u$ is the corporate income tax rate, $w$ and $z$ are the effective rate of investment tax credit and the present value of depreciation allowances, $\tau$ is the indirect tax rate, $i$ is a weighted average of debt and equity costs, and $\phi$ is the depreciation rate. These parameters are constructed from Bell System data whenever possible.

14. We can illustrate the problem of disentangling "excess capacity" and economies of scale which was discussed in Section 1 . The long run cost elasticity $n_{\ell}=\left(1+\pi^{*}\right)^{-1}$, evaluated at the static equilibrium levels of capital and $R \& D$, is $n_{\ell}=0.55$. If the observed levels are used instead, we get the short run cost elasticity, $n_{s}=0.45$. 
Note that since $n_{8}<n_{\ell}$, the incorrect use of observed levels would overstate the degree of scale economies. This reflects the "excess capacity" in capital reported in Table 4 (the underuse of R\&D is insufficient to counterbalance this effect).

15. Note two points. First, the values $1=.055$ and $g=.07$ are the average values for the Bell System during the sample period. Second, the parameter $\delta$ can be interpreted as follows. From the discussion preceding equation (21), $P_{t}-\bar{C}_{t}=\left(\partial \bar{C}_{0} / \partial z_{t}\right) e^{-\delta t}$, so $e^{-\delta t}$ is the fraction of the reduction in average cost which is not yet reflected in the price of output $t$ years later. The range of $\delta$ used in the text, .25 $\leq \delta \leq .35$, implies that 20-30 percent of the cost reduction is still not reflected in the price after five years.

16. For R\&D the estimate of $\rho$ varies inversely with $\delta$ and $\theta$ because $\rho>1$. For example, a higher value of $\delta$ means faster dissipation of the positive rents which accrue to R\&D. For capital, $\rho$ varies directly with $\delta$ and $\theta$ because $\rho<1$. Also see note 6 . 


\section{$\underline{\text { References }}$}

Appelbaum, E. "Testing Neoclassical Production Theory." Journal of Econometrics, 1978, 7(1), 87-102.

Berndt, E. R. and B. C. Fleld (eds.). Modeling and Measuring Natural

Resource Substitution. Cambridge, Mass.: The MIT Press, 1981.

Berndt, E. R. and M. A. Fuss. "Productivity Measurement Using Capital

Asset Valuation to Adjust for Variations in Utilization." National

Bureau of Economic Research, Working Paper No. 895, 1982.

Berndt, E., C. Morrison and G. Watkins. "Dynamic Models of Energy Demand:

An Assessment and Comparison. In E. R. Berndt and B. C. Fields

(eds.), Modeling and Measuring Natural Resource Substitution.

Cambridge, Mass.: The MIT Press, 1981.

Berndt, E. and N. E. Savin. "Estimation and Hypothesis Testing in

Singular Equation Systems with Auto-Regressive Disturbances."

Econometrica, $1975,43(5-6), 937-957$.

Borts, G. "The Estimation of Rall Cost Functions." Econometrica, 1960, $28,108-131$.

Brown, R. S. and L. R. Christensen. "Estimating Elasticities of Substitution in a Model of Partial Static Equilibrium: An Application to U.S. Agriculture, 1947 to 1974." In E. R. Berndt and B. C.

Fields (eds.), Modeling and Measuring Natural Resource Substitution. Cambridge, Mass.: The MIT Press, 1981.

Caves, D. W., L. R. Christensen, and J. A. Swanson. "Productivity Growth, Scale Economies, and Capacity Utilization in U.S. Railroads, 195574." American Economic Review, 1981, 71(5), 994-1002. 
Christensen, L., D. Cummings and P. Schoeck. "Econometric Estimation of Scale Economies in Telecommunications." Social Service Research Institute, University of Wisconsin, Madison, Working Paper 8013, 1980.

Courvilie, L. "Regulation and Efficiency in the Electric Utility Industry." Bell Journal of Economics and Management Science, 1974, $5(1), 53-74$.

Cowing, T. "The Effectiveness of Rate of Return Regulation: An Empirical Test Using Profit Functions." In M. Fuss and D. McFadden (eds.), Production Economics: A Dual Approach to Theory and Applications. Amsterdam: North-Holland, 1978.

Denny, M., M. Fuss and L. Waverman. "Substitution Possibilities for Energy: Evidence from U.S. and Canadian Manufacturing Industries." In E. R. Berndt and B. C. Field (eds.), Modeling and Measuring Natural Resource Substitution. Cambridge, Mass.: The MIT Press, 1981 .

Epstein, L. and M. Denny. "The Multivariate Flexible Accelerator Model:

Its Empirical Restrictions and an Application to U.S. Manufacturing." Econometrica, $1983,51(3), 647-674$.

Gorman, W. "Measuring the Quantities of Fixed Factors." In J. Wolfe (ed.), Value, Capital and Growth: Papers in Honour of Sir John Hicks. Chicago: Aldine Publishing Co., 1968.

Hanoch, G. "The Elasticity of Scale and the Shape of Average Costs." American Economic Review, 1975, 65, 956-965.

Hausman, J. A. "Specification Tests in Econometrics." Econometrica, $1978,46,1251-1272$. 
Holly, A. "A Remark on Hausman's Specification-Test.". Econometrica, $1982,50,749-759$.

Lau, L. J. "A Characterization of the Normalized Restricted Profit Function." Journal of Economic Theory, 1976, 12, 131-163.

Lau, L. and P. Yotopolous. "A Test for Relat1ve Efflclency and Application to Indian Agriculture." American Economic Review, $1971,61,94-109$.

Morrison, C. and E. Berndt. "Short-Run Labor Productivity in a Dynamic Model." Journal of Econometrics, $1981,15,339-365$.

Nadir1, M. I. and M. A. Schankerman. "The Structure of Production, Technological Change and the Rate of Growth of Total Factor Productivity in the Bell System." In T. Cowing and R. Stevenson (eds.), Productivity Measurement in Regulated Industries. New York: Academic Press, 1981a.

Nadir1, M. I. and M. Schankerman. "Techntcal Change, Returns to Scale, and the Productivity Slowdown." American Economic Review Papers and Proceedings, $1981 \mathrm{~b}, 71(2), 314-319$.

National Science Foundation. Research and Development in Industry. Washington, D.C.: U.S. Government Printing Office, annual.

Pakes, A. and M. Schankerman. "The Rate of Obsolescence of Patents, Research Gestation Lags, and the Private Rate of Return to Research Resources." In Z. Griliches (ed.), R\&D, Patents and Productivity. Chicago: The University of Chicago Press, 1984. Samuelson, P. "Prices of Factors and Goods In General Equilibrium." Review of Economic Studies, $1953-4,: 21,1-20$. 
Spann, R. "Rate of Return Regulation and Efficiency in Production: An Empirical Test of the Averch-Johnson Thesis." Bell Journal of Economics and Management Science, 1974, 5(1), 38-52.

Uzawa, H. "Production Functions with Constant Elasticities of Substitution." Review of Economic Studies, 1962, 29, 291-299. 